
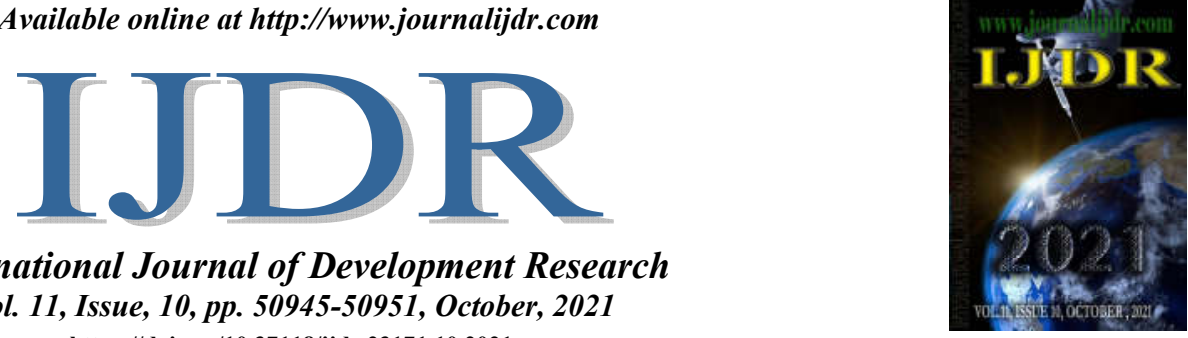

ISSN: 2230-9926

International Journal of Development Research

Vol. 11, Issue, 10, pp. 50945-50951, October, 2021

https://doi.org/10.37118/ijdr.23171.10.2021

\title{
AVALIAÇÃO DA INFLUÊNCIA DE INIBIDORES DE BOMBA DE PRÓTONS NA ABSORÇÃO DE VITAMINA B12: REVISÃO SISTEMÁTICA DA LITERATURA
}

\section{Anna Luisa Silva Campos ${ }^{1}$, Maria Clara de Angeli ${ }^{1}$, Felipe Bertollo Ferreira ${ }^{2}$, Ana Paula Hamer Sousa Clara ${ }^{3}$ and Maria das Graças Silva Mattede ${ }^{4}$}

${ }^{1}$ Acadêmicos de Medicina da Escola Superior de Ciências da Santa Casa de Misericórdia de Vitória (EMESCAM)

${ }^{2}$ Mestre, Santa Casa de Misericórdia de São Paulo (SCMSP) - Professor da EMESCAM, Serviço de gastroenterologista do Hospital Santa Casa de Misericórdia de Vitória (HSCMV)

${ }^{3}$ Mestre, Hospital Santa Casa de Misericórdia de Vitória (HSCMV) - Professora da EMESCAM, Serviço de gastroenterologista da Hospital Santa Casa de Misericórdia de Vitória (HSCMV)

${ }^{4}$ Doutora, Universidad Autónoma de Asunción (UAA) - Professora da EMESCAM/ Universidade Federal do Espírito Santo aposentada (UFES)

\section{ARTICLE INFO}

\section{Article History:}

Received $27^{\text {th }}$ August, 2021

Received in revised form

$20^{\text {th }}$ September, 2021

Accepted $19^{\text {th }}$ October, 2021

Published online $30^{\text {th }}$ October, 2021

Key Words:

Vitamina B12.

Deficiência de vitamina B12,

Omeprazol.

*Corresponding author:

Isadora Brito Lima

\begin{abstract}
Objetivo: Avaliar se o uso crônico de inibidores de bomba de prótons pode influenciar na absorção de vitamina B12 e, consequentemente, em seu nível sérico, por meio de uma revisão da literatura. Método: Revisão de literatura sistemática nas bases de dados do Pubmed e Cochrane no período de 28 anos, onde foram encontrados 256 artigos. Após a exclusão de 244 deles, foram analisados $12 \mathrm{em}$ sua totalidade. Foram considerados positivos os trabalhos que demonstraram: hipovitaminose B12, diminuição de vitamina B12 sérica, diminuição da absorção da vitamina ou aumento da chance de reposição de B12. Resultado e Discussão: Dos 12 trabalhos, $75 \%$ demonstraramuma associação positiva, mesmo com desfechos diferentes. Os outros estudos não encontraram associação positiva entre o uso do medicamento a nenhum desses parâmetros. Considerações finais: A análise sugere existir associação entre o uso crônico de IBP e a diminuição sérica de vitamina B12.
\end{abstract}

Copyright (C) 2021, Anna Luisa Silva Campos et al. This is an open access article distributed under the Creative Commons Attribution License, which permits unrestricted use, distribution, and reproduction in any medium, provided the original work is properly cited.

Citation: Anna Luisa Silva Campos, Maria Clara de Angeli, Felipe Bertollo Ferreira, Ana Paula Hamer Sousa Clara and Maria das Graças Silva Mattede. "Avaliação da influência de inibidores de bomba de prótons na absorção de vitamina b12: revisão sistemática da literatura", International Journal of Development Research, 11, (10), 50945-50951.

\section{INTRODUCTION}

Os Inibidores da Bomba de Prótons (IBP) são a classe de medicamentos mais comumente usada para o tratamento de distúrbios relacionados à acidez gástrica (Wolfe, 2000). Eles atuam inibindo a bomba de prótons no estômago, nas células parietais, promovendo uma supressão gástrica e inibem eficazmente a secreção ácida basal e da estimulada, de maneira dose-dependente (Omeprazol). As condições nas quais os IBPs são mais eficazes e comumente usados são para o tratamento de doenças ácido-pépticas, alívio dos sintomas de pirose, regurgitação ácida e dor epigástrica.
São indicados para doença ulcerosa péptica, doença do refluxo gastroesofágico (DRGE), lesões gastrointestinais causadas por antiinflamatórios não esteroidais (AINEs), dispepsias, condições patológicas hipersecretoras incluindo síndrome de Zollinger-Ellison, erradicação da bactéria Helicobacter pylori em associação a um tratamento antibacteriano, prevenção de ressangramento de úlceras gastroduodenais, estenoses esofágicas e terapia de manutenção do esôfago de Barrett (Esomeprazol magnésio; Mukherjee, 2018; Arai, 2011). Desde sua produção pela indústria farmacêutica, na década de 1980, essa classe de medicamentos vem sendo amplamente utilizada em pacientes de todo o mundo, substituindo procedimentos médicos, incluindo a cirurgia de úlcera péptica (Arai, 2011). Porém, nos últimos anos os IBPs têm sido submetidos a estudos sobre efeitos e 
interações adversas, entre eles a deficiência de vitamina B12, despertando cautela e cuidados entre os profissionais médicos sobre a indicação para determinados pacientes. Sendo assim, o objetivo desta revisão é avaliar a influência do uso crônico e contínuo de inibidores de bomba de prótons no organismo humano, sobretudo na absorção de vitamina $\mathrm{B} 12$.

\section{METODOLOGIA}

Foi realizada uma revisão sistemática da literatura com objetivo de verificar a associação do uso crônico de inibidores de bombas de prótons e a diminuição do nível sérico de vitamina B12. Foram utilizados os seguintes termos para pesquisa: "proton, pump, inhibitors OR, PPI OR, omeprazole OR, lansoprazole OR, esomeprazole OR, pantoprazole OR, drug-induced OR, antiulcer AND $B 12$ vitamin $O R, B 12$ vitamin deficiency OR, cobalamin NOT child", nas bases de dados PubMed e Cochrane, sem limite de tempo (estudo mais antigo de 1992) e em inglês. Os artigos encontrados foram sendo excluídos após a análise dos títulos e posteriormente dos resumos. Por fim, os artigos remanescentes ao final dessasanálises preliminares foram estudados em sua totalidade.

\section{Critérios de exclusão}

- Trabalhos em outras línguas senão inglês;

- Trabalhos duplicados;

- Trabalhos que focavam em outro desfecho senão absorção de vitamina B12;

- Trabalhos que focavam em outra classe medicamentosa senão IBPs;

- Trabalhos de revisão ou meta-análise.

De acordo com o fluxograma de Prisma (Figura 1), foram encontrados 256 artigos, que foram sendo excluídos até chegar aos 12 trabalhos analisados na íntegra, sendo eles descritivos ou intervencionistas, entre os quais 6 de caso controle, 3 de coorte prospectivo, 1 de coorte retrospectivo, 1 ensaio clínico e 1 misto.

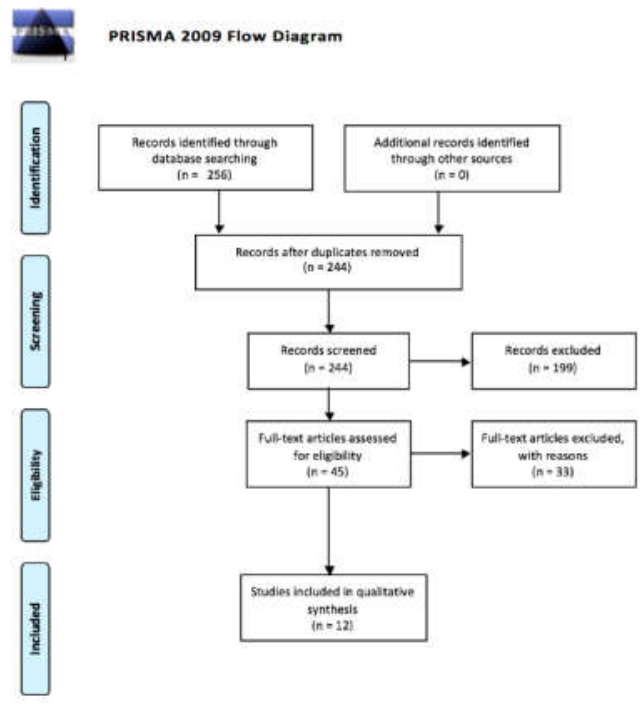

Fonte: os autores, 2020.

Figura 1. Prisma 2009 FlowDiagram

\section{REVISÃO DE LITERATURA}

O estômago é um importante órgão do aparelho gastrointestinal que tem como principal função a digestão e, em menor intensidade, a absorção das substâncias deglutidas.

Para isso, o órgão secreta várias substâncias, incluindo o ácido clorídrico $(\mathrm{HCl})$, gastrina, renina, pepsina e histamina. ${ }^{6}$ Este órgão é revestido por quatro camadas: mucosa, submucosa, muscular e serosa.
A maioria dos elementos funcionais secretores está na camada mucosa que é constituída pelo epitélio colunar simples. Essas células epiteliais produzem grande quantidade de muco e bicarbonato os quais são os principais responsáveis pela cito proteção contra o ácido, pepsinas, substâncias químicas e outros. O epitélio mucoso se invagina formando criptas que conferem acesso das glândulas gástricas à superfície luminal. Morfofuncionalmente, pode-se diferenciar este revestimento em 3 regiões:

- $\quad 1^{\mathrm{a}}$ região (Cárdia): Região de transição entre o epitélio escamoso do esôfago e a mucosa gástrica;

- $\quad 2^{\mathrm{a}}$ região (Seguimento secretor de ácido): Anatomicamente, se localiza no fundo e no corpo gástrico e é formada pelas glândulas oxínticas (parietais ou fúndicas). Nestas glândulas encontramos as células parietais, principais (ou pépticas), endócrinas e indiferenciadas;

- $\quad 3^{\mathrm{a}}$ região (Piloro): Anatomicamente, se localiza no antro e no piloro e é constituído por glândulas pilóricas.

Já se tem conhecimento de que a mucosa gástrica é a camada secretora mais importante e são as células parietais, presentes nas glândulas oxínticas, as responsáveis por esta função. Elas produzem o ácido clorídrico, os fatores intrínsecos e a maioria das enzimas gástricas. Tais células são estimuladas por alguns fatores, como a acetilcolina, a gastrina e a histamina, sendo que esta última se liga a receptores de histamina $\mathrm{H} 2$, ativando a bomba de prótons SódioPotássio-ATPase ( $\mathrm{Na}+\mathrm{K}+\mathrm{ATPase})$ liberando assim o ácido clorídrico (HCl). (Bighetti, 2002) Como exposto acima, os IBPs representam uma classe de medicamentos muito utilizadosem todo o mundo para o tratamento de distúrbios relacionados à acidez gástrica, ${ }^{1}$ uma vez que, ao inibir a bomba de íons supracitada da célula gástrica parietal, promovem a supressão gástrica, e ainda de maneira mais eficaz que os antagonistas de receptores H2 (outra classe semelhante, que também visa à redução da acidez gástrica, porém através da via da histamina). ${ }^{2,5}$ Entre os medicamentos que a compõem, podemos citar: omeprazol, lansoprazol, pantoprazol, pantoprazol magnésico, pantoprazol sódico, rabeprazol, esomeprazol e dexlansoprazol. ${ }^{5}$ Eles são manipulados com diversas apresentações de formas farmacêuticas: $O$ omeprazol possui a forma de apresentação de cápsula dura de liberação retardada, o pantoprazol comprimido revestido, e o esomeprazol comprimido revestido de liberação retardada, sendo a maioria de 20,30 ou 40 miligramas (mg) (Omeprazol; Esomeprazol magnésio; Pantoprazol).

O mecanismo de ação é complexo e se dá de maneira que os medicamentos são ativados em meio ácido nas células parietais do estômago e, primeiramente, ativam as bombas de NA+K+ATPase, com as quais formam uma ligação covalente, inibindo-as de maneira irreversível. No entanto, sua meia vida é curta e novas bombas são sintetizadas a todo o momento, sendo necessárias novas doses do medicamento de 24 em 24 horas para efeito farmacocinético. Como exemplo, pode-se evidenciar a biodisponibilidade do pantoprazol, que atinge concentrações plasmáticas máximas em aproximadamente 2 horas a 2 horas e 30 minutos após a sua administração, passando então a decair em nível plasmático após este tempo, sendo, portanto, recomendada uma nova dose do medicamento após $24 \mathrm{~h}$ para manter o $\mathrm{pH}$ do estômago em níveis alcalinos satisfatórios (Omeprazol; Pantoprazol). Nos últimos anos, interações adversas e efeitos colaterais têm sido relatados pelos pacientes resultando cuidados para sua indicação. Algumas das reações adversas do uso prolongado de IBP incluem osteoporose com risco aumentado de fraturas ósseas, diarreia associada a Clostridium difficile, pneumonia, hipomagnesemia, deficiência de vitamina B12, deficiência de ferro, nefrite intersticial aguda, hipergastrinemia e gastrite atrófica crônica (McColl, 2009; Freston, 1994; Kuipers, 2006). Um estudo recente avaliou a ocorrência de encefalopatia hepática $(\mathrm{EH})$ em um grupo de cirróticos em uso de IBP (análise prospectiva por uma média de 14,1 meses) e mostrou que na população que fez usodo medicamento houve desenvolvimento de $\mathrm{EH}$ de forma evidentemente maior comparado ao grupo que não fez ( $64 \%$ vs $25 \%)$ e sobrevida global menor ( $41 \%$ vs $81 \%$ ). Ou seja, houveum aumento significativo de casos de encefalopatia hepática, além do risco de morte durante o 
segmento do estudo, independentemente de fatores prognósticos da cirrose como idade eModelo para Doença Hepática Terminal (MELD) (Nardelli, 2019). Além disso, um outro estudo mostrou ainda o aumento da mortalidade entre pacientes tratados com IPBs por longo tempo: Estimatesofall cause mortalityand cause specific mortality associated with próton pump inhibitors among US veterans: cohortstudy (Xie, 2019). Segundo os autores, há evidência de que o uso prolongado de IBP é associado a eventos adversos graves, sendo mais prevalentes com tempo de uso mais longo. $\mathrm{O}$ estudo acompanhou através de coorte prospectivo um grupo de usuários de IBPs (tabela 1).

Outro estudo de inibidores $\mathrm{H} 2$ por um período de 10 anos, comprovou um risco aumentado de mortalidade no grupo que usou IBP por todas as causas testadas por eles (tabela 2), principalmente por doença cardiovascular, doença renal crônica e câncer gastrointestinal superior. Houve mais mortes no geral entre os pacientes em uso de IBP do que naqueles em uso de bloqueadores H2 (37,92\% e 35,69\%, respectivamente) e o tempo até a morte foi menor no primeiro grupo (4,84 e 4,96 anos, respectivamente). Conclui-se então, no artigo, que esses medicamentos devem ser usados somente quando indicados e pelo tempo mínimo necessário.

Deficiência de vitamina B12: As deficiências vitamínicas são um problema comum em todo o mundo, sendo a hipovitaminose B12 uma delas. Essa deficiência, em particular, pode levar a transtornos hematológicos, neurológicos e cardiovasculares por interferir, principalmente, no metabolismo da homocisteína (Hcy) e nas reações de metilação do organismo.15 Essa vitamina não é sintetizada pelo organismo humano, tendo sua fonte natural em alimentos de origem animal, como leite, carne e ovos. Sua deficiência é muito frequente, além das pessoas com hipocloridria gástrica, entre idosos e vegetarianos. Seu diagnóstico pode ser dado, de acordo com a maioria das referências, pelo nível de cobalamina sérica menor que $200 \mathrm{pg} / \mathrm{ml}$ (148pmol/l), mas também pelo aumento dos níveis de ácido metilmalônico (MMA) e Hcy. 15-17 Alguns autores recomendam solicitar esses dois exames quando o valor da vitamina é intermediário (entre 100 e $400 \mathrm{pg} / \mathrm{ml}$ ) para comprovar a deficiência, como mostra a Figura 2.

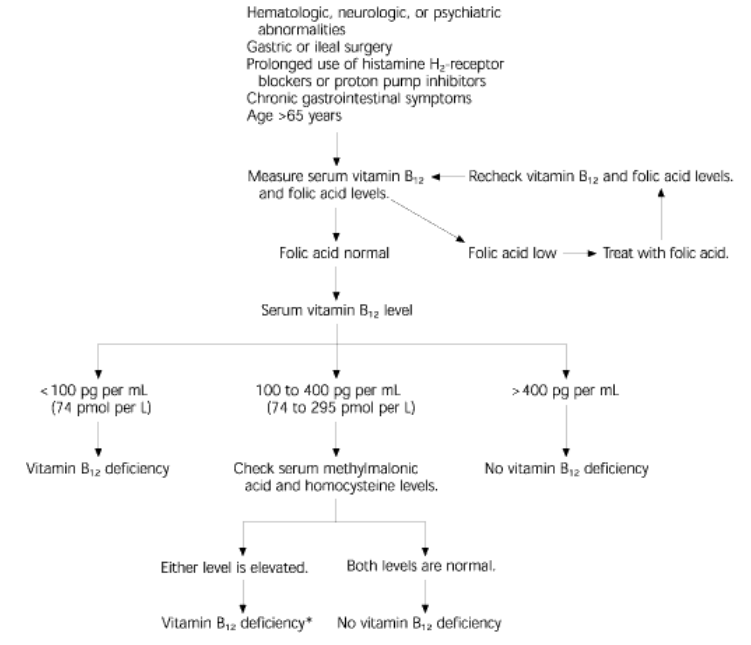

Fonte: Oh RC, Brown DL. Vitamin B12 deficiency. American Family Physician. 2003. Acesso em: 07/05/2020.

\section{Figura 2. Interpretação da deficiência de vitamina B12}

Com relação à sua absorção no organismo humano (Figura 3), a vitamina B12 é capturada pela transcobalamina $\mathrm{I}$, uma proteína $\mathrm{R}$ produzida na saliva e no estômago. Na presença de $\mathrm{pH}$ ácido e de enzimas proteolíticas pancreáticas (tripsina), ocorre a liberação da cobalamina dessa proteína salivar e a transferência da molécula para o fator intrínseco gástrico (FI), produzido pelas células parietais do estômago. ${ }^{15} \mathrm{Na}$ mucosa, o complexo formado pela ligação da vitamina B12 ao FI é capaz de resistir às enzimas proteolíticas da luz intestinal e percorrer todo o trato gastrointestinal, até chegar ao íleo terminal, onde adere-se a receptores específicos e é absorvida. 15 Sendo assim, entende-se que o aumento do $\mathrm{pH}$ do estômago pode levar a uma dificuldade na ligação da cobalamina ao FI e, consequentemente, sua absorção fica prejudicada, o que ao longo do tempo pode levar a uma deficiência vitamínica.

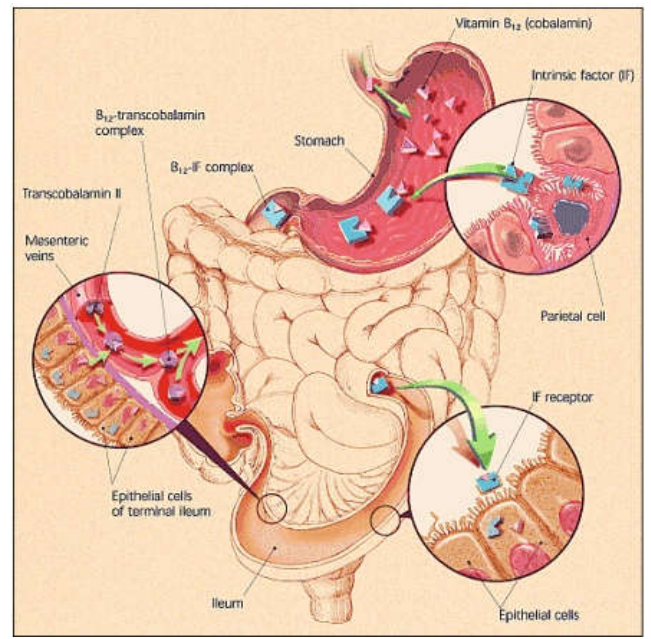

Fonte: Oh RC, Brown DL. Vitamin B12 deficiency. American Family Physician. 2003. Acesso em: 07/05/2020.

\section{Figura 3 - Mecanismo de absorção da vitamina B12}

A causa mais comum hoje em dia de hipovitaminose B12 é a má absorção de cobalamina ligada ao alimento. Um fator comum a muitas das condições que podem levar a má absorção é a incapacidade de liberar vitamina B12 da proteína R. Como exemplo, podemos citar a acloridria, gastrite, gastrectomia e uso de inibidores da bomba de prótons ou outros antiácidos, os quais resultam em diminuição da secreção de ácido clorídrico, reduzindo assim a liberação de vitamina B12 das proteínas alimentares. 16, 17

\section{RESULTADOS}

Dos 12 trabalhos analisados, 75\% (9) apontaram para uma associação positiva, sendo que 3 associaram o uso de Inibidores de Bomba de Prótons com a hipovitaminose B12, 2 com a diminuição de vitamina B12 sérica, 2 com a diminuição da absorção de vitamina B12 e 2 com o aumento da chance de reposição de B12 (como descreve a figura 4). Sendo assim, os outros $25 \%$ não encontraram associação positiva entre o uso do medicamento a nenhum desses parâmetros.

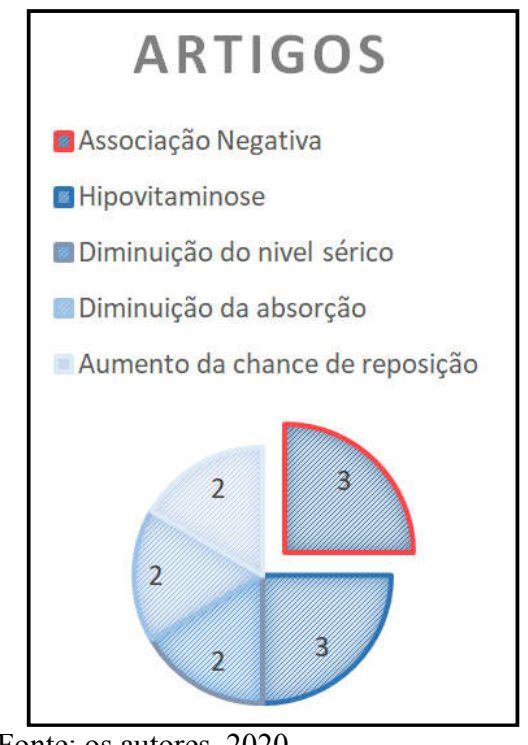

Fonte: os autores, 2020.

Figura 4. Gráfico de análises dos resultados dos artigos 


\section{Quadro 1. Relação de títulos e autores considerados na análise}

\section{Título}

Proton pump inhibitor and histamine2 receptor antagonist use and vitamin B12 deficiency.

Vitamin B12 deficiency in hypersecretors during long-term acid suppression with próton pump inhibitors.

A case-control study on adverse effects: $\mathrm{H} 2$ blocker or próton pump inhibitor use and risk of vitamin B12 deficiency in older adults.

Effect oflong-term gastric acid suppressive therapy on sérum vitamin B12 levels in patients with Zollinger-Ellison syndrome.

Effect of short- and long-term treatment with omeprazole on the absorption and sérum levels of cobalamin.

Effect of hypochlorhydria due to omeprazole treatment or atrophic gastrites on protein-bound vitamin B12 absorption.

Omeprazole therapy causes malabsorption of cyanocobalamin (vitamin B12)

Serum iron, ferritin, and vitamin B12 during prolonged omeprazole therapy. The association between antiulcer medication and initiation of cobalamin replacement in older persons.

Use of próton pump inhibitors is not associated withVitamin B12 deficiency and in older hospital patients: A case control study

Increased Vitamin B12 Requirement Associated with Chronic Acid Suppression Therapy

Vitamin B12 Deficiency is Linked with Long-Term Use of Proton Pump Inhibitors in Institutionalized Older Adults: Could a Cyanocobalamin Nasal Spray be Beneficial?

Fonte: os autores, 2020 .

\section{Autores}

Jameson R. Lam, MPH; Jennifer L. Schneider, MPH;WeiZhao, MPH; Douglas A. Corley, MD, PhD B. I. HIRSCHOWITZ, J. WORTHINGTON \& J. MOHNEN

Robert J. Valuck e J. Mark Ruscin

Basel Termanini, MD, FathiaGibril, MD, Vincent E. Sutliff, MD, FangYu, MD, David J. Venzon, PhD, Robert T. Jensen, MD

B. E. SCHENK, H. P. M. FESTEN*, E. J. KUIPERS, E. C. KLINKENBERG-KNOL \& S. G. M. MEUWISSEN

John R. Saltzman, MD, James A. Kemp, MD, Barbara B. Golner, RN, Marcos C. Pedrosa, MD, Gerard E. Dallai, MD, and Robert M. Russell, MD, FACN

Stefan P. Marcuard; Lisa Albernaz; andPrabhaker G. Khazanie

Herbert Koop, M.D., and Max Georg Bachem, M.D.

Susan L. Mitchella, *, Kenneth Rockwood

P.E. Cotter, S.T. O'Keeffe

Rex W Force, Angela D Meeker, Paul S Cady, Vaughn L Culbertson, Wendy S Force, and Craig M Kelley

Nancy R. Rozgony MS, RD, LD a ,ChengshunFang $\mathrm{PhD}$ a , Marie F. Kuczmarski PhD a \& Harold Bob $\mathrm{MD}$

\section{Quadro 2. Quadro de análises comparativas}

\begin{tabular}{|c|c|c|c|c|}
\hline Título & Tipo de estudo & Resultado & $\begin{array}{l}\text { Número de pacientes } \\
\text { usando IBP }\end{array}$ & Diagnostico de hipovitaminose \\
\hline $\begin{array}{l}\text { Proton pump inhibitor and histamine } 2 \text { receptor } \\
\text { antagonist use and vitamin B12 deficiency. }\end{array}$ & Caso controle & $\begin{array}{l}\text { Associação } \\
\text { positiva }\end{array}$ & 16330 & nível sérico $<281 \mathrm{pg} / \mathrm{ml}$ \\
\hline $\begin{array}{l}\text { Vitamin B12 deficiency in hypersecretors during long- } \\
\text { term acid suppression with próton pump inhibitors. } \\
\text { A case-control study on adverse effects: } \mathrm{H} 2 \text { blocker or } \\
\text { próton pump inhibitor use and risk of vitamin B12 } \\
\text { deficiency in older adults. }\end{array}$ & $\begin{array}{l}\text { Coorte prospectivo } \\
\text { Caso controle }\end{array}$ & $\begin{array}{l}\text { Associação } \\
\text { positiva } \\
\text { Associação } \\
\text { positiva }\end{array}$ & 53 & $\begin{array}{l}\text { nível sérico, Hcy e MMA (não } \\
\text { cita valores) } \\
<130 \mathrm{pg} / \mathrm{mL} \text { ou entre } 130- \\
300 \mathrm{pg} / \mathrm{mL} \text { com MMA }>271 \\
\text { nmol/1 e HCYS }>13.9 \text { umol } / 1\end{array}$ \\
\hline $\begin{array}{l}\text { Effect oflong-term gastric acid suppressive therapy on } \\
\text { sérum vitamin B12 levels in patients with Zollinger- } \\
\text { Ellison syndrome. }\end{array}$ & Coorte prospectivo & $\begin{array}{l}\text { Associação } \\
\text { positiva }\end{array}$ & 111 & nível sérico $<200 \mathrm{pg} / \mathrm{mL}$ \\
\hline $\begin{array}{l}\text { Effect of short- and long-term treatment with } \\
\text { omeprazole on the absorption and sérum levels of } \\
\text { cobalamin. }\end{array}$ & $\begin{array}{l}\text { Grupo 1: ensaio clinico } \\
\text { Grupo } 2: \quad \text { coorte } \\
\text { histórico }\end{array}$ & $\begin{array}{l}\text { Associação } \\
\text { negativa }\end{array}$ & 33 & nível sérico $<180 \mathrm{pg} / \mathrm{mL}$ \\
\hline $\begin{array}{l}\text { Effect of hypochlorhydria due to omeprazole treatment } \\
\text { or atrophic gastrites on protein-bound vitamin B12 } \\
\text { absorption. }\end{array}$ & Coorte prospectivo & $\begin{array}{l}\text { Associação } \\
\text { positiva }\end{array}$ & 8 & $\begin{array}{l}\text { pelo nível sérico, mas não } \\
\text { relata valor }\end{array}$ \\
\hline $\begin{array}{l}\text { Omeprazole therapy causes malabsorption of } \\
\text { cyanocobalamin (vitamin B12) }\end{array}$ & Ensaio clínico & $\begin{array}{l}\text { Associação } \\
\text { positiva }\end{array}$ & 10 & $\begin{array}{l}\text { pelo nível sérico, mas não } \\
\text { relata valor }\end{array}$ \\
\hline $\begin{array}{l}\text { Serum iron, ferritin, and vitamin B12 during prolonged } \\
\text { omeprazole therapy. }\end{array}$ & Coorte retrospectivo & $\begin{array}{l}\text { Associação } \\
\text { negativa }\end{array}$ & 34 & $\begin{array}{l}\text { pelo nível sérico, mas não } \\
\text { relata valor }\end{array}$ \\
\hline $\begin{array}{l}\text { The association between antiulcer medication and } \\
\text { initiation of cobalamin replacement in older persons. }\end{array}$ & Caso controle & $\begin{array}{l}\text { Associação } \\
\text { positiva }\end{array}$ & 125 & $\begin{array}{l}\text { não houve análise (usou as } \\
\text { pessoas que tiveram que repor } \\
\text { a vitamina) }\end{array}$ \\
\hline $\begin{array}{l}\text { Use of próton pump inhibitors is not associated } \\
\text { withVitamin B12 deficiency and in older hospital } \\
\text { patients: A case control study }\end{array}$ & Caso controle & $\begin{array}{l}\text { Associação } \\
\text { negativa }\end{array}$ & 59 & nível sérico $<150 \mathrm{pmol} / 1$ \\
\hline $\begin{array}{l}\text { Increased Vitamin B12 Requirement Associated with } \\
\text { Chronic Acid Suppression Therapy }\end{array}$ & Caso controle & $\begin{array}{l}\text { Associação } \\
\text { positiva }\end{array}$ & 125 & $\begin{array}{l}\text { não houve análise (usou as } \\
\text { pessoas que tiveram que repor } \\
\text { a vitamina) }\end{array}$ \\
\hline $\begin{array}{l}\text { Vitamin B12 Deficiency is Linked with Long-Term Use } \\
\text { of Proton Pump Inhibitors in Institutionalized Older } \\
\text { Adults: Could a Cyanocobalamin Nasal Spray be } \\
\text { Beneficial? }\end{array}$ & Caso controle & $\begin{array}{l}\text { Associação } \\
\text { positiva }\end{array}$ & 17 & nível sérico $<350 \mathrm{pmol} / 1$ \\
\hline
\end{tabular}

Fonte: os autores, 2020. 
Somando-se os 9 trabalhos que apresentaram associação entre o uso de IBPs e os desfechos em questão, o número de pacientes acompanhados foi de 16.840 , o número de dias de acompanhamento foi de 20640,5 e a média de acompanhamento de 2.293 dias $(6,28$ anos). Enquanto isso, os 3 trabalhos que não encontraram associação somaram um número total de pacientes acompanhados de 93, número de dias acompanhados de 2.079 e média de acompanhamento de 1.040 (2,84 anos). A maioria dos trabalhos foi realizada nos Estados Unidos (8 dos 12), 1 foi na Holanda, 1 na Alemanha, 1 no Canadá e 1 no Reino Unido. Com relação ao ano de publicação, 4 foram publicados a partir de 2010, 4 entre 2000 e 2009 e outros 4 antes de 2000, sendo o mais antigo de 1992. Em relação à quantificação da vitamina $\mathrm{B} 12$ no organismo, 10 não citaram qualquer informação relacionada ao nível sérico de vitamina B12 antes do uso de IBP para posterior comparação e apenas 2 possuíam controle da vitamina B12 antes de ser usado IBP. Nenhum trabalho utilizou na amostra pacientes abaixo de 18 anos. Três trabalhos utilizaram pacientes entre 18 e 60 anos (equivalente a 104 pacientes) e 6 utilizaram com mais de 60 anos (387 pacientes). Além disso, 1 trabalho não cita a idade de seus pacientes ( 34 ao todo) e 2 usaram pacientes acima de 18 anos sem especificar a faixa etária (16.441 pacientes). O trabalho Proton pump inhibit or and histamine 2 receptor antagonist use and vitamin B12 deficiency não discriminou a faixa etária acima de 18 anos e corresponde a um valor de 16.330 pacientes. Com relação ao tempo de acompanhamento, houve uma média de dias acompanhados de 2.065 dias, com desvio padrão amostral de \pm 2.209 . Com isso, pode-se dizer que o tempo de acompanhamento dos pacientes em cada estudo foi altamente variável, uma vez que um trabalho acompanhou durante 14 dias e outro acompanhou por 6.570 (os de menor e maior acompanhamento, respectivamente), e por esse motivo fez-se a mediana, que foi de 1.714 dias.

Entretanto, o trabalho Proton pump inhibitor and histamine 2 receptor antagonist use and vitamin $B 12$ deficiency teve muita influência nesses números, uma vez que a mediana sem ele foi de 1.222 dias (ele representa $22,49 \%$ de todos os trabalhos). Dentre os 12 trabalhos, a média do número de dias de acompanhamento de paciente foi de 7.678.561, com um desvio padrão de \pm 101.788 . A mediana foi de 56.562, com uma variação absoluta de 140 a 83.446.300 pacientes dia. Mais uma vez, o mesmo trabalho citado anteriormente teve uma influência muito grande, sendo que representou $98,79 \%$ de todos os trabalhos, sendo a média sem este trabalho de 101.788 e a mediana de 46.950 . Com relação ao uso de IBP pelas populações estudadas, a média de pacientes que usou IBP nos trabalhos foi de 1.413. Porém, a variação absoluta foi muito grande, variando de 8 a 16330 pacientes, sendo que a mediana foi de 56 pacientes. Novamente, $96,25 \%$ dos pacientes são referentes ao trabalho de maior impacto (sem a análise desse trabalho a média seria de 57 pacientes). Entretanto, nenhum dos trabalhos controlou rigidamente a dose de IBP. Dos 12 estudos, 6 não realizaram nenhum controle sobre a dose do IBP e, dos outros 6, 1 controlou com o número de comprimidos por dia, mas não a miligramagem. Os outros 5 descreveram a miligramagem da medicação, que variou de 20 a 120 $\mathrm{mg}$ de omeprazol e 15 a $450 \mathrm{mg}$ de lansoprazol, no entanto, não estabeleceram a quantidade que os pacientes deveriam tomar. Apenas 4 estudos fizeram o controle de acidez nos pacientes da população e, ainda assim, 50\% destes o fizeram com pacientes com síndrome de Zollinger-Ellison na amostra e os outros $50 \%$ citaram, porém não demonstraram esse controle. Dos 12 artigos, 1 obteve o diagnóstico de hipovitaminose pelos prontuários dos pacientes, 9 obtiveram por dosagem direta de nível séricoe 2 pela necessidade de reposição de vitamina B12. Dos que usaram a dosagem direta do nível sérico, 2 fizeram dosagem de Homocisteína e Ácido Metil Malônico, o que aumentou a taxa de detecção da hipovitaminose em pelo menos $30 \%$. Todos os trabalhos e seus autores estão contemplados no Quadro 1, e comparações pertinentes à análise foram descritos no Quadro 2.

\section{DISCUSSÃO}

$\mathrm{Na}$ literatura existe uma grande quantidade de trabalhos que buscam relacionar o uso prolongado de inibidor da bomba de próton a seus efeitos colaterais, incluindo câncer gástrico, infecções bacterianas, hipergastrinemia, pneumonias, deficiências vitamínicas e osteoporose. ${ }^{5}$ Essa revisão tentou basear-se em trabalhos que discutissem estritamente sobre a absorção de vitamina B12, relacionando esse desfecho ao uso de inibidores de bombas de prótons. Nesse trabalho, analisou-se 12 estudos de diferentes qualidades, sendo distribuídos entre casos controle, coortes prospectivos, coortes retrospectivos e ensaios clínicos. Essa distribuição foi semelhante a outras revisões bibliográficas, como Association between vitamin B12 deficiency and long-term use of acid-lowering agents: a systematic review and meta-analysis e DrugInduced Vitamin B12 Deficiency: A Focus on Proton Pump Inhibitors and Histamine-2 Antagonists, as quais também encontraram, ao final de suas análises, uma associação positiva entre o uso de IBP e a hipovitaminose B12. Vale a pena pontuar, ainda, que os três trabalhos possuem como fontes principais trabalhos de caso-controle, o que reforça a adequabilidade e replicabilidade dessa pesquisa. Dentre os 12 trabalhos analisados, $75 \%$ aponta uma associação positiva, sugerindo veracidade na hipótese inicial, o que também se assemelha ao observado em outras revisões citadas anteriormente. Dos três trabalhos que não encontraram associação positiva, nenhum possui relevância similar àqueles que encontraram, uma vez que os somando, o número total de pacientes acompanhados foi de 93 e a média de acompanhamento de 2,85 anos, contra 16.840 pacientes e média de 6,28 anos nos estudos que encontraram associação.

Para se realizar um estudo retrospectivo é fundamental que haja credibilidade nos dados de registros a serem computados, em relação à exposição do fator e à sua intensidade, assim como pela ocorrência da doença/situação clínica por esse motivo. ${ }^{18}$ No caso dessa revisão, trabalhos que busquem o uso prévio de IBP em pacientes já diagnosticados com a deficiência vitamínica são considerados ideais para a análise que buscamos. Sendo assim, é benéfico que haja estudos de caso controle, uma vez que é o melhor para identificar fatores de risco/etiologia em desfechos raros e que demandem longo tempo para ocorrer, como é o caso da hipovitaminose B12 em decorrência de uso prévio de IBPs. Portanto, tem-se como ponto positivo que metade das análises são do tipo caso-controle. A diminuição nos níveis de vitamina B12 pode estar associada, independentemente do uso de IBP, ao envelhecimento. Em idosos, a hipovitaminose pode ser associada a fatores encontrados mais comumente nessa faixa etária, como anemia perniciosa, cirurgias gástricas e atrofia gástrica, que levam à má absorção da vitamina em questão. ${ }^{19}$ Portanto, é interessante que os estudos possuam amostras de diferentes faixas etárias, o que foi avaliado nos trabalhos que descreveram a idade dos pacientes. Além disso, é ideal para a revisão que se saiba o valor prévio do nível sérico de vitamina B12 das populações para entender por meio de comparação o impacto que o IBP teve sobre esse nível. Dos artigos, apenas 2 possuíam controle de tal vitamina antes de ser usado IBP e a maioria não cita informações em relação a isso. No artigo Effect of long-term gastric acid suppressive therapy on sérum vitamin B12 levels in patients with Zollinger-Ellison syndrome, esse dado fazia parte apenas de uma subanálise do estudo, com 8 pacientes e, mesmo assim, não apresentava comparação com grupo controle e nem diferenciava o uso de IBP e inibidores H2. Já no artigo Effect of short- and long-term treatment with omeprazole on the absorption and sérum levels of cobalamin, houve a comparação da vitamina B12 antes e depois do uso do IBP em todos os pacientes da amostra, porém foi utilizado para isso a excreção da vitamina B12, e não os níveis séricos.

O tempo de acompanhamento é outro fator de grande relevância para avaliação da relação entre o uso de IBP e a hipovitaminose B12, levando-se em consideração que ela está associada ao uso crônico da medicação. ${ }^{20}$ Como mencionado, há grande variabilidade de dados na literatura nesse aspecto, e não há descrito um tempo específico necessário para provocar alterações proliferativas gástricas, ${ }^{5}$ porém, sabe-se que um período de acompanhamento muito curto não é suficiente para desencadeá-lo em todos os pacientes. Dessa forma, consideramos o tempo médio de acompanhamento dos pacientes nos artigos analisados (média de 5,7 anos), satisfatório e compatível com o tema. 
Um ponto positivo foi que os trabalhos que encontraram associação positiva são aqueles com maior tempo de acompanhamento, e podemos citar como exemplo Proton pump inhibitor and histamine 2 receptor antagonist use and vitamin $B 12$ deficiency, que analisou os pacientes entre 1997 e 2011. Esse é um dos trabalhos com maior repercussão na literatura sobre o assunto e o com maior peso nessa análise (maior número de pacientes e maior tempo de acompanhamento) e também foi discutido em outras revisões como Association between vitamin B12 deficiency and long-term use of acid-lowering agents: a systematic review and meta-analysis e DrugInduced Vitamin B12 Deficiency: A Focus on Proton Pump Inhibitors and Histamine-2 Antagonists e ele possui um forte impacto em todas as categorias analisadas nesta revisão. Os autores compararam um grupo de 25.956 pacientes que já possuíam diagnóstico de deficiência de vitamina B12 contra um de 184.199 que não possuíam e analisaram o uso pregresso de IBPs e anti-histamínicos H2. É um trabalho de caso controle, o que é bom para a interpretação do desfecho como discutido anteriormente, embora esse tipo de estudo esteja sujeito a vieses, principalmente de seleção. ${ }^{18}$ No caso do trabalho em questão, os pesquisadores se basearam em bancos de dados e utilizaram um grupo controle com características distintas às dos casos, o que nos faz refletir sobre a aplicabilidade do trabalho para diferentes populações.

As deficiências significativas de vitamina B12 podem ser detectadas por aumento de ácido metilmalônico e homocisteína (MMA e Hcy), mesmo na ausência de danos hematológicos e sem a necessidade de baixos níveis de vitamina B12 sérica total. A associação de testes laboratoriais tem auxiliado a reconhecer e estudar estágios precoces, expandindo a definição de deficiência de vitamina B12. ${ }^{15}$ Sendo assim, vê-se que a análise da hipovitaminose B12 é débil em inúmeros artigos, uma vez que apenas dois deles utilizaram esses métodos, que possuem tamanha importância para o diagnóstico precoce. Além disso, os níveis de vitamina $\mathrm{B} 12$ séricos são considerados baixos quando sua concentração é inferior a $200 \mathrm{pg} / \mathrm{ml}$ (148pmol/l). ${ }^{16}$ Muitos estudos não citaram a dosagem que consideram como hipovitaminose e, dentre os que demonstraram a dosagem sérica, utilizaram valores entre 130 e $900 \mathrm{pg} / \mathrm{ml}$, ou seja, valoresdistintos dos descritos na literatura, e muito variáveis, o que pode ter interferido nos resultados. Como já mencionado, os inibidores de bombas de prótons são amplamente utilizados ao redor do mundo para tratamento de várias doençasem doses e tempo de tratamento diferentes. Eles podem causar múltiplos efeitos adversos dependentes de dose, além da hipovitaminose B12, como infecção por Clostridium difficile, fraturas, nefrite intersticial, pneumonia, eficácia reduzida do clopidogrel e reaparecimento mais acentuado (rebound) de hipersecreção ácida quando interrompidos. ${ }^{21}$ Por isso, é importante para a análise causal do uso em relação a esses efeitos que os estudos fornecessem a dose dos tratamentos. Porém, metade dos artigos não realizou nenhum controle sobre a dose do IBP e da outra metade, um controlou com o número de comprimidos por dia, mas não a miligramagem, e os outros 5 descreveram a miligramagem da medicação (que variou de 20 a $120 \mathrm{mg}$ de omeprazol e 15 a $450 \mathrm{mg}$ de lansoprazol), porém não estabeleceram a quantidade que os pacientes deveriam tomar por dia. Assim, conclui-se que as doses variaram muito em cada paciente e não houve rigor nesse controle na maioria dos estudos, o que é prejudicial para se formar uma conclusão causal.

Dois dos estudos analisados, ambos com associação positiva entre a hipovitaminose B12 e o uso dos inibidores de bombas de prótons, tiveram como amostra pacientes com a síndrome de Zollinger Ellison, o que pode ter alterado o desfecho em questão. Essa síndrome é caracterizada como hipergastrinemia decorrente de um gastrinoma, que leva ao aumento da acidez gástrica. ${ }^{22}$ Nesses casos, a supressão ácida pelo IBP deve ser feita de forma mais prolongada e em doses mais altas, o que pode promover quadro de má absorção e deficiência de vitamina B12 mais acentuados em pacientes com essa síndrome do que em outras populações, diminuindo a aplicabilidade desses estudos. ${ }^{23}$ Em suma, entende-se que a maioria dos trabalhos que existem na literatura mundial sobre o tema apontam para uma associação positiva entre o uso prolongado dos inibidores de bomba de prótons e a diminuição da absorção de cobalamina, o que pode ser explicado pela diminuição da secreção de ácido clorídrico e consequente diminuição da acidez gástrica. No entanto, destacamos alguns pontos negativos nos trabalhos, o que nos leva a analisá-los com parcimônia.

\section{CONSIDERAÇÕES FINAIS}

Diante da presente análise, considerou-se que os inibidores de bombas de prótons ainda permanecem como medicamentos essenciais para tratamentos na medicina atual, como doença ulcerosa péptica, doença do refluxogastroesofágico, dispepsias, erradicação da bactéria Helicobacter pylori e terapia de manutenção do esôfago de Barrett. Esse trabalho reforça que o uso crônico dos IBPs pode levar à diminuição da absorção de vitamina B12pelo sistema gastrointestinal. No entanto, espera-se que futuros trabalhos possam colaborar na demonstração de uma associação causal entre a exposição e o desfecho. Sendo assim, é de extrema importância que se continue estudando esse tema, visando à ampliação do conhecimento sobre o uso desses medicamentos levando à hipovitaminose B12.

\section{REFERÊNCIAS}

Alventosa-Mateu C, Ferrer-Barceló L, Huguet-Malavés JM, FerrerArranz L, Monzó-Gallego A, Medina-Chuliá E. Síndrome de Zollinger-Ellison. Rev Esp Enfermedades Dig. 2013;105(10):641-3.

Andrès E, Loukili N H, Noel E, Kaltenbach G, Ben Abdelgheni M, Perrin AE, et al. Vitamin B12 (cobalamin) deficiency in elderly patients. 2004; 171: 251-9.

Arai A, Maria S, Gallerani C. 2011. Uso Crônico De Fármacos Inibidores Da Bomba De Prótons: Eficácia Clínica e Efeitos Adversos.

Bighetti A E, Antônio MA, De Carvalho, JE. 2002. Regulação e Modulação Da Secreção Gástrica. Revista de Ciências Médicas, 11(1): 55-60.

Coussirat C. Prevalência de Deficiência De Vitamina B12 e Ácido Fólico e Sua Assoçiaçao com Anemia em Idosos Atendidos em Um Hospital Universitário. 2010;

Esomeprazol magnésio [Bula]. Ranbaxy Farmacêutica Ltda. .Esomeprazol Magnésico Comprimidos Revestidos $20 \mathrm{mg} \&$ $40 \mathrm{mg}$

FrestonJW. Omeprazole, hypergastrinemia, and gastric carcinoid tumors. Annals of Internal Medicine, 1994;121(3):232-3.

George HM. Supressão Ácida: Utilização dos Inibidores da Bomba de Protões e das suas Alternativas Terapêuticas. 2011;1-20.

Hochman B, Nahas FX, OliveiraRS F, Ferreira LM. Desenhos de pesquisa. Acta Cir Bras [Revista em Internet]. 2005; 2(9).

Hoefler R, Leite B F. Segurança do uso contínuo de inibidores da bomba de prótons. Bol Farmacoter. 2009;1 e 2:1-6.

Kuipers EJ. Proton pump inhibitors and gastric neoplasia. 2006; 55: 1217-21.

McColl K. Effect of Proton Pump Inhibitors on Vitamins and Iron. Am J Gastroenterol [Revista em Internet], 2009; 104.

Mukherjee S, Jana T, Pan J.J. 2018. Adverse Effects of Proton Pump Inhibitors on Platelet Count: A Case Report and Review of the Literature. Case Rep Gastrointest Med [ Revista em Internet], 15.

Nardelli S, Gioia S, Ridola L, Farcomeni A, Merli M, Riggio O. Proton Pump Inhibitors Are Associated With Minimal and Overt Hepatic Encephalopathy and Increased Mortality in Patients With Cirrhosis. Hepatology. 2019;

Omeprazol [Bula]. Brainfarma Indústria Química e Farmacêutica S.A. Cápsula Dura de Liberação Retardada $20 \mathrm{mg}$.

Paniz C, Grotto D, Schmitt GC, Valentini J, Schott KL, Pomblum VJ, et al. Fisiopatologia da deficiência de vitamina B12 e seu diagnóstico laboratorial. J Bras Patol e Med Lab [Revista em Internet]. 2005; 323-34.

Pantoprazol [Bula]. Accord Farmacêutica Ltda. Comprimido Gastrorresistente $20 \mathrm{mg}$ 
Pantoprazol [Bula]. Merck S / A.Comprimido revestido 20mg e Comprimido revestido $40 \mathrm{mg}$

Rodrigues S S, Fonseca, C C Neves, M, T, D. 2005. Células endócrinas do sistema gastroenteropancreático: Conceitos, distribuição, secreções, ação e controle. Arquivos de Ciências Veterinárias e Zoologia, 8 (2):171-180.

Sheen E, Triadafilopoulos G. Adverse effects of long-term proton pump inhibitor therapy. Digestive Diseases and Sciences 2011; 56: 931-50.
Shipton MJ, Thachil J. Vitamin B12 deficiency - A 21st century perspective. Clin Med. 2015;15(2):145-50.

Wolfe MM, Sachs G. 2000. Acid suppression: Optimizing therapy for gastroduodenal ulcer healing, gastroesophageal reflux disease, and stress-related erosive syndrome. Gastroenterology [Revista em Internet], 118(2): 9-31.

Xie Y, Bowe B, Yan Y, Xian H, Li T, Al-Aly Z. Estimates of all cause mortality and cause specific mortality associated with proton pump inhibitors among US veterans: Cohort study. BMJ. 2019. 